\title{
Solving Nonsmooth Equations Using Derivative-Free Methods
}

\author{
${ }^{1}$ M. S. M. Bahgat, ${ }^{2}$ M.A. Hafiz \\ ${ }^{1}$ Department of mathematics, Faculty of Science, Minia University, Minia 61111 Egypt. \\ ${ }^{2}$ Department of mathematics, Faculty of Science and arts, Najran University, 1988, Saudi Arabia.
}

Keywords: Nonlinear equations; Convergence analysis; Nonsmooth equations; Iterative methods; Derivative-free methods

\begin{abstract}
In this paper, a family of derivative-free methods of cubic convergence for solving nonlinear equations is suggested. In the proposed methods, several linear combinations of divided differences are used in order to get a good estimation of the derivative of the given function at the different steps of the iteration. The efficiency indices of the members of this family are equal to 1.442. The convergence and error analysis are given. Also, numerical examples are used to show the performance of the presented methods and to compare with other derivative-free methods. And, were applied these methods on smooth and nonsmooth equations.
\end{abstract}

\section{Introduction}

In recent years many researchers have developed several iterative methods for solving nonlinear equations. In this paper we are going to develop efficient methods to find approximations of the root $\mathrm{r}$ of $f(x)=0$ without evaluation of derivatives. A number of ways are considered by many researchers to improve the local order convergence of Newton's method by the expense of additional evaluations of the functions, derivatives and changes in the points of iterations see [14]. There are several different methods in literature for the computation of the root $r$ of the nonlinear equation $f(x)=0$ The most famous of these methods is the classical Newton's method (NM):

$$
x_{n+1}=x_{n}-\frac{f\left(x_{n}\right)}{f^{\prime}\left(x_{n}\right)} .
$$

which is a well-known basic method and converges quadratically in the neighbor-hood of simple root $r$. This method is not applicable when the derivative of any function is not defined in any interval. Therefore the Newton's method was modified by Steffensen, who replaced the first derivative $\mathrm{f}^{\prime}(\mathrm{x})$ in Newton's method by forward difference approximation

$$
f^{\prime}\left(x_{n}\right)=\frac{f\left(x_{n}+f\left(x_{n}\right)\right)-f\left(x_{n}\right)}{f\left(x_{n}\right)}=P_{0}\left(x_{n}\right) .
$$

and obtained the famous Steffensen's method (SM) [5] :

$$
x_{n+1}=x_{n}-\frac{f\left(x_{n}\right)^{2}}{f\left(x_{n}+f\left(x_{n}\right)\right)-f\left(x_{n}\right)} .
$$

which is the known Steffensen's method (SM). Newton and Steffensen's methods are of second order converges, both require two functional evaluations per step, but in contrast to Newton's method, Steffensen's method is free from any derivative of the function, because sometimes the applications of the iteration methods which depend upon derivatives are restricted in engineering. A family of Steffensen like methods was derived in [6-9] free from derivatives, which uses three functional evaluations per step and has cubic convergence. Recently, Cordero et al. [10] proposed a derivative free iterative method by replacing the forward-difference approximation in Ostrowski's method by the central-difference approximation. However, it is still a method of third order and requires four functional evaluations per iteration. Therefore these methods have efficiency index $3^{1 / 4} \approx 1.1316$ which is less than $2^{1 / 2} \approx 1.4142$ of the Newton and Steffensen [11]. 
The rest of the paper is organized as follows: in Section 2 we describe our family of methods and we show the order of convergence of it. In Section 3, different numerical test confirm the theoretical results and allow us to compare this family with other known methods mentioned in this section.

\section{Description of the methods}

For the sake completeness, we recall the methods in [10], [12] and [4], these methods respectively as follows:

Algorithm 2.1. For a given $x_{0}$, compute approximates solution $x_{n+1}$ by the iterative schemes

$$
\begin{aligned}
& y_{n}=x_{n}-\frac{f\left(x_{n}\right)}{f^{\prime}\left(x_{n}\right)}, \\
& x_{n+1}=y_{n}-\frac{f\left(x_{n}\right)^{2}+f\left(y_{n}\right)^{2}}{f^{\prime}\left(x_{n}\right)\left(f\left(x_{n}\right)-f\left(y_{n}\right)\right)} .
\end{aligned}
$$

Algorithm 2.1 has fourth-order convergence, which was obtained by [13]. Homeier [3] derived the following cubically convergent iteration scheme.

Algorithm 2.2. For a given $\mathrm{x}_{0}$, compute approximates solution $x_{n+1}$ by the iterative scheme

$$
x_{n+1}=x_{n}-\frac{2 f\left(x_{n}\right) f^{\prime}\left(x_{n}\right)}{2 f^{\prime 2}\left(x_{n}\right)-f^{\prime}\left(x_{n}\right) f^{\prime \prime}\left(x_{n}\right)} .
$$

Algorithm 2.3. For a given $\mathrm{x}_{0}$, compute approximates solution $x_{n+1}$ by the iterative schemes

$$
\begin{gathered}
y_{n}=x_{n}-\frac{f\left(x_{n}\right)}{f^{\prime}\left(x_{n}\right)}, \\
x_{n+1}=x_{n}-\frac{2 f\left(x_{n}\right)}{f^{\prime}\left(x_{n}\right)+f^{\prime}\left(y_{n}\right)} .
\end{gathered}
$$

The first and the second derivatives with respect to, y which may create some problems. To overcome this drawback, several authors have developed involving only the first derivatives. This idea plays a significant part in developing our new iterative methods free from first and second derivatives with respect to y.To be more precise, we now approximate $f^{\prime}\left(y_{n}\right)$ to reduce the number of evaluations per iteration by a combination of already known data in the past steps. Toward this end, an estimation of the function $P_{1}(t)$ is taken into consideration as follows

$$
\begin{aligned}
& P_{1}(t)=a+b\left(t-x_{n}\right)+c\left(t-x_{n}\right)^{2} \\
& P_{1}^{\prime}(t)=b+2 c\left(t-x_{n}\right)
\end{aligned}
$$

By substituting in the known values

$$
\begin{aligned}
& P_{1}\left(y_{n}\right)=f\left(y_{n}\right)=a+b\left(y_{n}-x_{n}\right)+c\left(y_{n}-x_{n}\right)^{2} \\
& P_{1}^{\prime}\left(y_{n}\right)=f^{\prime}\left(y_{n}\right)=b+2 c\left(y_{n}-x_{n}\right) \\
& P_{1}\left(x_{n}\right)=f\left(x_{n}\right)=a \\
& P_{1}^{\prime}\left(x_{n}\right)=f^{\prime}\left(x_{n}\right)=b
\end{aligned}
$$

we could easily obtain the unknown parameters. Thus we have

$$
f^{\prime}\left(y_{n}\right)=2\left(\frac{f\left(y_{n}\right)-f\left(x_{n}\right)}{y_{n}-x_{n}}\right)-f^{\prime}\left(x_{n}\right)=P_{1}\left(x_{n}, y_{n}\right)
$$


At this time, it is necessary to approximate $f^{\prime \prime}\left(y_{n}\right)$ with a combination of known values Accordingly, we take account of an interpolating polynomial

$$
P_{2}(t)=a+b\left(t-x_{n}\right)+c\left(t-x_{n}\right)^{2}+d\left(t-x_{n}\right)^{3}
$$

and also consider that this approximation polynomial satisfies the interpolation conditions

$$
f\left(x_{n}\right)=P_{2}\left(x_{n}\right), \quad f\left(y_{n}\right)=P_{2}\left(y_{n}\right), \quad f^{\prime}\left(x_{n}\right)=P_{2}^{\prime}\left(x_{n}\right)
$$

And $f^{\prime}\left(y_{n}\right)=P_{2}^{\prime}\left(y_{n}\right)$ By substituting the known values in $P_{2}(t)$ we have a system of three linear equations with three unknowns. By solving this system and simplifying we have

$$
f^{\prime \prime}\left(y_{n}\right)=\frac{2}{y_{n}-x_{n}}\left(\frac{f\left(y_{n}\right)-f\left(x_{n}\right)}{y_{n}-x_{n}}-f^{\prime}\left(x_{n}\right)\right)=P_{2}\left(x_{n}, y_{n}\right) \text {. }
$$

Using (1) we can also remove the first derivative from (2) and (3)

$$
\begin{gathered}
f^{\prime}\left(y_{n}\right)=2\left(\frac{f\left(y_{n}\right)-f\left(x_{n}\right)}{y_{n}-x_{n}}\right)-\frac{f\left(x_{n}+f\left(x_{n}\right)\right)-f\left(x_{n}\right)}{f\left(x_{n}\right)}=P_{1}\left(x_{n}, y_{n}\right) \\
f^{\prime \prime}\left(y_{n}\right)=\frac{2}{y_{n}-x_{n}}\left(\frac{f\left(y_{n}\right)-f\left(x_{n}\right)}{y_{n}-x_{n}}-\frac{f\left(x_{n}+f\left(x_{n}\right)\right)-f\left(x_{n}\right)}{f\left(x_{n}\right)}\right)=P_{2}\left(x_{n}, y_{n}\right) .
\end{gathered}
$$

Now using the equations (1), (2) and (3) to suggest the following new iterative methods for solving nonlinear equation, It is established that the following new methods have convergence order three, which will denote by Bahgat Hafiz Methods (BHM1-BHM5).Then algorithm 2.1 can be written in the following form.

BHM1: For a given $x_{0}$, compute approximates solution $x_{n+1}$ by the iterative schemes

$$
\begin{gathered}
y_{n}=x_{n}-\frac{f\left(x_{n}\right)^{2}}{f\left(x_{n}+f\left(x_{n}\right)\right)-f\left(x_{n}\right)}, \\
x_{n+1}=x_{n}-\frac{f\left(x_{n}\right)^{2}+f\left(y_{n}\right)^{2}}{P_{0}\left(x_{n}\right)\left(f\left(x_{n}\right)-f^{\prime}\left(y_{n}\right)\right)} .
\end{gathered}
$$

We use Steffensen's method as predictor and Algorithm 2.2 as a corrector then we have the following new methods have convergence order three.

BHM2: For a given $x_{0}$, compute approximates solution $x_{n+1}$ by the iterative schemes

$$
\begin{gathered}
y_{n}=x_{n}-\frac{f\left(x_{n}\right)^{2}}{f\left(x_{n}+f\left(x_{n}\right)\right)-f\left(x_{n}\right)}, \\
x_{n+1}=y_{n}-\frac{2 f\left(y_{n}\right) P_{1}\left(x_{n}, y_{n}\right)}{2 P_{1}^{2}\left(x_{n}, y_{n}\right)-f\left(y_{n}\right) P_{2}\left(x_{n}, y_{n}\right)} .
\end{gathered}
$$

BHM2 is called the new two-step modified Halley's method free from second and first derivative, for solving nonlinear equation $f(x)=0$ 
BHM3: For a given $x_{0}$, compute approximates solution $x_{n+1}$ by the iterative schemes

$$
\begin{gathered}
y_{n}=x_{n}-\frac{f\left(x_{n}\right)^{2}}{f\left(x_{n}+f\left(x_{n}\right)\right)-f\left(x_{n}\right)}, \\
x_{n+1}=x_{n}-\frac{2 f\left(x_{n}\right)}{P_{0}\left(x_{n}\right)+P_{1}\left(x_{n}, y_{n}\right)} .
\end{gathered}
$$

BHM3 is called the new two-step modified Homeier's method free from derivatives, for solving nonlinear equation $f(x)=0$. Now we can modified the methods in [14] by removing the derivatives in the following two methods.

BHM4: For a given $x_{0}$, compute approximates solution $x_{n+1}$ by the iterative schemes

$$
\begin{gathered}
y_{n}=x_{n}-\frac{f\left(x_{n}\right)^{2}}{f\left(x_{n}+f\left(x_{n}\right)\right)-f\left(x_{n}\right)}, \\
x_{n+1}=y_{n}+\frac{f\left(y_{n}\right)}{P_{0}\left(x_{n}\right)}-\frac{2 f\left(x_{n}\right) f\left(y_{n}\right)}{P_{0}\left(x_{n}\right)\left(f\left(x_{n}\right)-f\left(y_{n}\right)\right)} .
\end{gathered}
$$

BHM5: For a given $x_{0}$, compute approximates solution $x_{n+1}$ by the iterative schemes

$$
\begin{gathered}
y_{n}=x_{n}-\frac{f\left(x_{n}\right)^{2}}{f\left(x_{n}+f\left(x_{n}\right)\right)-f\left(x_{n}\right)}, \\
x_{n+1}=y_{n}+\frac{f\left(y_{n}\right)}{P_{0}\left(x_{n}\right)}-\frac{4 f\left(y_{n}\right)}{P_{0}\left(x_{n}\right)+P_{1}\left(x_{n}, y_{n}\right)}
\end{gathered}
$$

Let us remark that, in terms of computational cost, the developed methods require only three functional evaluations per step. So, they have efficiency indices $3^{1 / 3} \approx 1.442$ which is higher than $2^{1 / 2} \approx 1.4142$ of the Steffensen's [11].

\section{Convergence analysis}

Let us now discuss the convergence analysis of the above mentioned algorithms.

Theorem 3.1: Let $r$ be a sample zero of sufficient differentiable function $f: \subseteq R \rightarrow R$ for an open interval $I$. If $x_{0}$ is sufficiently close to $r$, then the two step method defined by BHM2 has third-order convergence

Proof. Consider to

$$
\begin{gathered}
y_{n}=x_{n}-\frac{f\left(x_{n}\right)^{2}}{f\left(x_{n}+f\left(x_{n}\right)\right)-f\left(x_{n}\right)}, \\
x_{n+1}=y_{n}-\frac{2 f\left(y_{n}\right) P_{1}\left(x_{n}, y_{n}\right)}{2 P_{1}^{2}\left(x_{n}, y_{n}\right)-f\left(y_{n}\right) P_{2}\left(x_{n}, y_{n}\right)} .
\end{gathered}
$$

Let $r$ be a simple zero of $f$. Since $f$ is sufficiently differentiable, by expanding $f\left(x_{n}\right)$ about $r$, we get

$$
f\left(x_{n}\right)=c_{1} e_{n}+c_{2} e_{n}^{2}+c_{3} e_{n}^{3}+c_{4} e_{n}^{4}+O\left(e^{5}\right) .
$$


Furthermore, we have

$$
f\left(x_{n}\right)^{2}=c_{1}^{2} e_{n}^{2}+2 c_{1} c_{2} e_{n}^{3}+\left(2 c_{3} c_{1}+c_{2}^{2}\right) e_{n}^{4}+O\left(e_{n}^{5}\right) .
$$

Again by using Taylor's expansion we can get

$$
\begin{aligned}
f\left(x_{n}+f\left(x_{n}\right)\right) & =c_{1}\left(1+c_{1}\right) e_{n}+\left(3 c_{2} c_{1}+c_{2}+c_{1}^{2} c_{2}\right) e_{n}^{2} \\
& +\left(4 c_{3} c_{1}+2 c_{2}^{2}+2 c_{2}^{2} c_{1}+c_{3}+3 c_{3} c_{1}^{2}+c_{3} c_{1}^{3}\right) e_{n}^{3}+O\left(e^{4}\right)
\end{aligned}
$$

which gives

$$
\begin{aligned}
f\left(x_{n}+f\left(x_{n}\right)\right) & -f\left(x_{n}\right)=c_{1}^{2} e_{n}+\left(3 c_{2} c_{1}+c_{1}^{2} c_{2}\right) e_{n}^{2} \\
+ & \left(4 c_{3} c_{1}+2 c_{2}^{2}+2 c_{2}^{2} c_{1}+3 c_{3} c_{1}^{2}+c_{3} c_{1}^{3}\right) e_{n}^{3}+O\left(e^{4}\right)
\end{aligned}
$$

where $c_{k}=\frac{f^{(k)}(r)}{k !}, k=1,2,3, \ldots$ and $e_{n}=x_{n}-r$.

Now by substituting (9) and (11) in (6), we have

$$
y_{n}=r+\left(c_{2}+\frac{c_{2}}{c_{1}}\right) e_{n}^{2}+\left(2 \frac{c_{3}}{c_{1}}+c_{3} c_{1}-c_{2}^{2}+3 c_{3}-2 \frac{c_{2}^{2}}{c_{1}^{2}}\right) e_{n}^{3}+O\left(e^{4}\right) .
$$

By using Taylor's theorem, we have

$$
f\left(y_{n}\right)=c_{2}\left(1+c_{1}\right) e_{n}^{2}+\left(2 c_{3} c_{1}+c_{3} c_{1}^{3}-c_{2}^{2} c_{1}^{2}+3 c_{3} c_{1}^{2}-2 c_{2}^{2}-2 c_{2}^{2} c_{1}\right) \frac{e_{n}^{3}}{c_{1}}+O\left(e^{4}\right) \text {. }
$$

and

$$
\begin{gathered}
P_{1}\left(x_{n}, y_{n}\right)=c_{1}-c_{1} c_{2} e_{n}+\left(-c_{3}+c_{2}^{2}+2 \frac{c_{2}^{2}}{c_{1}}-c_{3} c_{1}^{2}-3 c_{1} c_{3}\right) e_{n}^{2}+O\left(e^{3}\right) \\
P_{2}\left(x_{n}, y_{n}\right)=2\left(c_{1} c_{2}+c_{2}\right)+\left(4 c_{3}-\frac{c_{2}^{2}}{c_{1}}+2 c_{3} c_{1}^{2}+6 c_{1} c_{3}+c_{1} c_{2}^{2}+2 c_{2}^{2}\right) e_{n}+O\left(e_{n}^{2}\right) .
\end{gathered}
$$

From (13), (14) and (15), we obtain

$$
\begin{gathered}
\frac{f\left(y_{n}\right)}{P_{1}\left(x_{n}, y_{n}\right)}=\frac{c_{2}}{c_{1}}\left(1+c_{1}\right) e_{n}^{2}+\left(-c_{2}^{2}+2 c_{3}+c_{3} c_{1}^{2}+3 c_{3} c_{1}-2 \frac{c_{2}^{2}}{c_{1}}-2 c_{2}^{2}\right) \frac{e_{n}^{3}}{c_{1}}+O\left(e_{n}^{4}\right) . \\
\frac{P_{2}\left(x_{n}, y_{n}\right)}{P_{1}\left(x_{n}, y_{n}\right)}=2\left(c_{2}+\frac{c_{2}}{c_{1}}\right)+\left(3 c_{2}^{2}+4 \frac{c_{2}^{2}}{c_{1}}+4 \frac{c_{3}}{c_{1}}-\frac{c_{2}^{2}}{c_{1}^{2}}+2 c_{3} c_{1}+6 c_{3}\right) e_{n}+O\left(e_{n}^{2}\right) . \\
\frac{1}{2} \frac{f\left(y_{n}\right)}{P_{1}\left(x_{n}, y_{n}\right)} \frac{P_{2}\left(x_{n}, y_{n}\right)}{P_{1}\left(x_{n}, y_{n}\right)}=\left(\frac{c_{2}^{2}}{c_{1}^{2}}+2 \frac{c_{2}^{2}}{c_{1}}+c_{2}^{2}\right) e^{2}+O\left(e^{3}\right)
\end{gathered}
$$

Then the equation (7) can be written as

$$
x_{n+1}=y_{n}-\frac{f\left(y_{n}\right)}{P_{1}\left(x_{n}, y_{n}\right)}\left[1-\frac{1}{2} \cdot \frac{f\left(y_{n}\right)}{P_{1}\left(x_{n}, y_{n}\right)} \cdot \frac{P_{2}\left(x_{n}, y_{n}\right)}{P_{1}\left(x_{n}, y_{n}\right)}\right]^{-1} .
$$

Now by substituting (16) and (18) in (19), we have

$$
x_{n+1}=r+\left(1-c_{1}\right) \frac{c_{2}^{2}}{c_{1}} e_{n}^{3}+O\left(e_{n}^{4}\right)
$$

From (20) and $e_{n+1}=x_{n+1}-r$, we have:

which shows (BHM2) has third-order convergence.

$$
e_{n+1}=\left(1-c_{1}\right) \frac{c_{2}^{2}}{c_{1}} e_{n}^{3}+O\left(e_{n}^{4}\right)
$$


Theorem 3.2: Let $r$ be a sample zero of sufficient differentiable function $f: \subseteq R \rightarrow R$ for an open interval $I$. If $x_{0}$ is sufficiently close to $r$, then the two- step method defined by BHM4 has third-order convergence

Proof. Consider to

$$
\begin{gathered}
y_{n}=x_{n}-\frac{f\left(x_{n}\right)^{2}}{f\left(x_{n}+f\left(x_{n}\right)\right)-f\left(x_{n}\right)}, \\
x_{n+1}=y_{n}+\frac{f\left(y_{n}\right)}{P_{0}\left(x_{n}\right)}-\frac{2 f^{\prime}\left(x_{n}\right) f\left(y_{n}\right)}{P_{0}\left(x_{n}\right)\left(f\left(x_{n}\right)-f\left(y_{n}\right)\right)} .
\end{gathered}
$$

Let $r$ be a simple zero of $f$. Since $f$ is sufficiently differentiable, by expanding $f\left(x_{n}\right), f\left(x_{n}+f\left(x_{n}\right.\right.$ ) ) and $f\left(y_{n}\right)$ about $r$ as in the theorem 3.1 we get

$$
\begin{gathered}
\frac{f^{\prime}\left(y_{n}\right)}{P_{0}\left(x_{n}\right)}=\frac{c_{2}}{c_{1}}\left(1+c_{1}\right) e_{n}^{2}+\left(2 \frac{c_{3}}{c_{1}}+c_{3} c_{1}-2 c_{2}^{2}+3 c_{3}-4 \frac{c_{2}^{2}}{c_{1}^{2}}-5 \frac{c_{2}^{2}}{c_{1}}\right) e_{n}^{3}+O\left(e_{n}^{4}\right) \\
f\left(x_{n}\right) f\left(y_{n}\right)=c_{1} c_{2}\left(1+c_{1}\right) e_{n}^{3}+O\left(e^{4}\right) \\
P_{0}\left(x_{n}\right)\left(f\left(x_{n}\right)-f\left(y_{n}\right)\right)=c_{1}^{2} e_{n}+2 c_{1} c_{2} e^{2}+\left(2 c_{1} c_{3}+c_{1} c_{2}^{2}+2 c_{2}^{2}\right) e_{n}^{3}+O\left(e_{n}^{4}\right)
\end{gathered}
$$

Now by substituting (12), (23), (24) and (25) in (22), we have

$$
x_{n+1}=r-\left(c_{2}^{2}+\frac{c_{2}^{2}}{c_{1}}\right) e_{n}^{3}+O\left(e_{n}^{4}\right)
$$

From (26) and $e_{n+1}=x_{n+1}-r$, we have:

$$
e_{n+1}=-\left(1+c_{1}\right) \frac{c_{2}^{2}}{c_{1}} e_{n}^{3}+O\left(e_{n}^{4}\right)
$$

from which it follows that (BHM4) has third-order convergence. In Similar way, we observe that the methods BHM1, BHM3 and BHM5 have also third order convergence as follows

$$
\begin{aligned}
& e_{n+1}=-\left(1+c_{1}\right) \frac{c_{2}^{2}}{c_{1}} e_{n}^{3}+O\left(e_{n}^{4}\right) \\
& e_{n+1}=-\left(c_{2}+\frac{c_{2}^{2}}{c_{1}}\right) e_{n}^{3}+O\left(e_{n}^{4}\right)
\end{aligned}
$$

\section{Numerical examples}

For comparisons, we have used the third-order Soleymani method (SM) [15] defined by

$$
\begin{gathered}
y_{n}=x_{n}-\frac{f\left(x_{n}\right)^{2}}{f\left(x_{n}+f\left(x_{n}\right)\right)-f\left(x_{n}\right)}, \\
x_{n+1}=x_{n}-\left[1+\frac{f\left(y_{n}\right)}{f\left(x_{n}\right)}\left(1+\frac{2 f\left(y_{n}\right)}{f\left(x_{n}\right)}\right)\right] \frac{f\left(y_{n}\right)}{P_{0}\left(x_{n}\right)} .
\end{gathered}
$$


Dehghan method (DM) [8]

$$
\begin{aligned}
& y_{n}=x_{n}-\frac{f\left(x_{n}\right)^{2}}{f\left(x_{n}+f\left(x_{n}\right)\right)-f\left(x_{n}\right)}, \\
& x_{n+1}=x_{n}-\frac{f\left(x_{n}\right)\left[f^{\prime}\left(x_{n}\right)+f\left(y_{n}\right)\right]}{f\left(x_{n}+f\left(x_{n}\right)\right)-f\left(x_{n}\right)} .
\end{aligned}
$$

and Jain method (JM) [6]

$$
\begin{gathered}
y_{n}=x_{n}-\frac{f\left(x_{n}\right)^{2}}{f\left(x_{n}+f\left(x_{n}\right)\right)-f\left(x_{n}\right)}, \\
x_{n+1}=x_{n}-\frac{f\left(x_{n}\right)^{3}}{\left[f\left(x_{n}+f\left(x_{n}\right)\right)-f\left(x_{n}\right)\right]\left[f\left(x_{n}\right)-f\left(y_{n}\right)\right]} .
\end{gathered}
$$

We consider here some numerical examples to demonstrate the performance of the new modified two-step iterative methods, namely algorithms (BHM1) - (BHM5). We compare the Soleymani method (SM), the Dehghan method (DM), Jain method (JM) and the new modified two-step methods algorithms (BHM1) - (BHM5), in this paper. In the Table 1 our examples are tested with precision $\varepsilon=10^{-15}$. All the computations are performed using Maple 15. The following examples are used for numerical testing:

Table 1: Test functions and their roots.

\begin{tabular}{ll}
\hline Functions & Roots \\
\hline$f_{1}(x)=\sin ^{2} x-x^{2}+1$, & 1.40449164821534 \\
$f_{2}(x)=x^{2}-e^{x}-3 x+2$, & 0.25753028543986 \\
$f_{3}(x)=\cos x-x$, & 0.73908513321516 \\
$f_{4}(x)=x-3 \log x$, & 1.85718386020784 \\
$f_{5}(x)=e^{-x}+\cos x$, & 1.74613953040801 \\
$f_{6}(x)=x-e \sin x-\mu$ & 0.38997777494636 \\
$f_{7}(x)=\frac{1}{x}-|x|$, & 1 \\
$f_{8}(x)=\left|x^{2}-9\right|$ & $-3,3$ \\
$f_{9}(x)= \begin{cases}x(x+1), & \text { if } x \leq 0, \\
-2 x(x-1), \text { if } x>0, & -1,0,1\end{cases}$ \\
$f_{10}(x)= \begin{cases}10\left(x^{4}+x\right), & \text { if } x<0, \\
-10\left(x^{3}+x\right), & \text { if } x \geq 0,\end{cases}$
\end{tabular}

where the function $f_{6}$ is Kepler's equation; $0 \leq \mathrm{e}<1$ and $0 \leq \mu \leq \pi$. We take values $\mu=0.01$ and $e=0.9995$. Furthermore, functions $f_{7} f_{10}$ are nonsmooth functions. In the Table 2, we listed the number of iterations for various methods, where the 'Div.' in the following tables imply that the method diverges (or the method terminates due to overflow). 
Table 2: Comparison of Number of iterations for various methods

\begin{tabular}{cllllllllll}
\hline Iterations & $f_{i}$ & $f_{2}$ & $f_{3}$ & $f_{4}$ & $f_{5}$ & $f_{6}$ & $f_{7}$ & $f_{8}$ & $f_{9}$ & $f_{i 0}$ \\
$x_{0}$ & 1.3 & 1.0 & 1.7 & 0.5 & 3.0 & 1.0 & -0.5 & 3.2 & 0.1 & -0.8 \\
\hline SM & 3 & 3 & 4 & 7 & 3 & 4 & 4 & Div. & 2 & 14 \\
DM & 3 & 3 & 3 & 7 & 3 & 5 & 3 & 3 & 2 & 25 \\
JM & 3 & 3 & 3 & 5 & 3 & 5 & 4 & 3 & 9 & 8 \\
BHM1 & 3 & 3 & 4 & 6 & 3 & 4 & 4 & 3 & 4 & 11 \\
BHM2 & 3 & 4 & 4 & 5 & 3 & 10 & 4 & 812 & 82 & 20 \\
BHM3 & 3 & 3 & 3 & 5 & 3 & 5 & 4 & 3 & 9 & 8 \\
BHM4 & 3 & 3 & 4 & 6 & 3 & 4 & 4 & 7 & 4 & 11 \\
BHM5 & 3 & 3 & 4 & 6 & 3 & 4 & 4 & 7 & 4 & 11 \\
\hline
\end{tabular}

Results are summarized in Table 2 as it shows, new algorithms are comparable with all of the methods and in most cases gives better or equal results.

\section{Conclusions}

The present study suggested a family of new derivative-free iterative methods for solving nonlinear equations. The efficiency indices of the members of this family are equal to 1.442 . In addition, these methods are derivative-free, which allow us to apply them also on nonsmooth equations with positive and promising results. Furthermore, these methods are particularly suited to those problems in which derivatives require lengthy. In the sequel, numerical examples have used in order to show the efficiency and accuracy of the novel methods from our suggested derivative-free class. Finally, it should be noted that, like all other iterative methods, the new methods from the class (BHM1)- (BHM5) have their own domains of validity and in certain circumstances should not be used.

\section{References:}

[1] M. Frontini, E. Sormani, Modified Newtons method with third-order convergence and multiple roots, J. Comput. Appl. Math. 156(2003) 345-354.

[2] H.H.H. Homeier, A modified Newton method for root finding with cubic convergence, J. Comput. Appl. Math. 157(2003) 227-230.

[3] H.H.H. Homeier, On Newton-type methods with cubic convergence, J. Comput. Appl. Math. $176(2005)$ 425-432.

[4] M. S. M. Bahgat, New Two-Step Iterative Methods for Solving Nonlinear Equations, J. Math. Research, 4, No. 3; June (2012) ISSN 1916-9795.

[5] D. Kincaid, W. Cheney, Numerical Analysis, second ed., Brooks/Cole, Pacific Grove, CA, (1996).

[6] P. Jain, Steffensen type methods for solving nonlinear equations, Applied Mathematics and Computation 194(2007) 527-533.

[7] Q. Zheng, J. Wang, P. Zhao, L. Zhang, A Steffensen-like method and its higher-order variants, Applied Mathematics and Computation 214(2009)10-16.

[8] M. Dehghan, M. Hajarian, Some derivative free quadratic and cubic convergence iterative formulas for solving nonlinear equations, Journal of Computational and Applied Mathematics 29(2010) 19- 30.

[9] N. Yasmin, M.Junjua, Some Drivative Free Iterative Methods for Solving nonlinear Equations. Academic Research International, Vol. 2, No. 1, (2012), 75-82. 
[10] A. Cordero, J.L. Hueso, E. Martínez, J.R. Torregrosa, Steffensen type methods for solving nonlinear equations J. of Comput and App Math 236 (2012) 3058-3064.

[11] J. M. Ortega, W. G. Rheinboldt, Iterative solutions of nonlinear equations in several variables, Press, New York- London, 1970.

[12] E. Halley, Anew exact and easy method for finding the roots of equations generally and without any previous reduction, Phil. Roy. Soc. London 8(1964) 136-147.

[13] K. Jisheng,L. Yitian, W. Xiuhua, A composite fourth-order iterative method for solving, Applied Mathematics and Computation (2006), doi:10.1016/j.amc.2006.05.181.

[14] R. Ezzati and F. Saleki, On the Construction of New Iterative Methods with Fourth-Order Convergence by Combining Previous Methods. International Mathematical Forum, Vol. 6, 2011, no. $27,1319-1326$

[15] F. Soleymani , V. Hosseinabadi, New Third- and Sixth-Order Derivative-Free Techniques for Nonlinear Equations. Journal of Mathematics Research 3 (2011) ISSN 1916-9809 (Online) 